\title{
Frequent Versus Infrequent Monitoring of Endotracheal Tube Cuff Pressures
}

\author{
Adam Letvin MD, Pamala Kremer RN CIC, Patty C Silver RRT MEd, \\ Nizama Samih RRT RRT-ACCS, Peggy Reed-Watts RRT MSc, and Marin H Kollef MD
}

\begin{abstract}
BACKGROUND: Currently there is no accepted standard of practice for the optimal frequency of endotracheal tube cuff pressure monitoring in mechanically ventilated patients. Therefore, we conducted a study to compare infrequent endotracheal tube cuff pressure monitoring (immediately after intubation and when clinically indicated for an observed air leak or due to tube migration) with frequent endotracheal tube cuff pressure monitoring (immediately after intubation, every $8 \mathrm{~h}$, and when clinically indicated). METHODS: We performed a prospective clinical trial with subjects assigned to study groups based on room assignment. The primary outcome was the occurrence of a ventilator-associated event (VAE) and was adjudicated by individuals blinded to the conduct of this study. RESULTS: We enrolled 305 subjects, with 166 (54.4\%) assigned to frequent monitoring and $139(\mathbf{4 5 . 6 \%})$ assigned to infrequent monitoring. The total number of endotracheal tube cuff pressure monitoring events for both groups was 1,531 versus 336, respectively. The occurrence of VAEs was infrequent and similar for both groups $(3.6 \%$ vs $5.8 \%, P=.37)$. Witnessed aspiration events $(0.6 \%$ vs $0 \%, P=.36)$, ventilator-associated pneumonia $(0 \%$ vs $0.7 \%, P=.27)$, 30-d mortality $(31.3 \%$ vs $30.2 \%, P=.83)$, and hospital length of stay $(10 \mathrm{~d}[6 \mathrm{~d}, 21 \mathrm{~d}]$ vs $11 \mathrm{~d}$ [6 d, 21 d], $P=.34$ ) were also similar for both study groups. The 30-d hospital readmission rate was statistically lower for the group that received infrequent monitoring $(15.1 \%$ vs $6.5 \%, P=.02)$. CONCLUSIONS: More frequent cuff pressure monitoring was not associated with any identifiable clinical outcome benefit. Key words: endotracheal tube; mechanical ventilation; ventilator-associated event. [Respir Care 2018;63(5):495-501. () 2018 Daedalus Enterprises]
\end{abstract}

\section{Introduction}

Currently there is no accepted standard for the frequency of monitoring endotracheal tube cuff pressure, and widely

\footnotetext{
Dr Letvin and Kollef are affiliatd with the Division of Pulmonary and Critical Care Medicine, Washington University School of Medicine, St Louis, Missouri; Ms Kremer is affiliated with the Hospital Epidemiology and Infection Prevention Department, Barnes-Jewish Hospital, St Louis, Missouri; Ms Silver, Ms Samih, and Ms Reed-Watts are affiliated with Respiratory Care Services, Barnes-Jewish Hospital, St Louis, Missouri.

Dr Kollef has disclosed a relationship with the Barnes-Jewish Hospital Foundation. The other authors have reported no conflicts of interests.

Correspondence: Marin H Kollef MD, Division of Pulmonary and Critical Care Medicine, Washington University School of Medicine, 4523 Clayton Ave, Campus Box 8052, St Louis, MO 63110. E-mail: kollefm@wustl.edu.
}

DOI: $10.4187 /$ respcare. 05926 different strategies are reported to include continuous monitoring. ${ }^{1}$ The implied goal of endotracheal tube cuff pressure monitoring is to maintain the cuff pressures between $20-30 \mathrm{~cm} \mathrm{H}_{2} \mathrm{O}$ to minimize air leaks and loss of tidal volume due to underfilling the cuff and to prevent injury to the tracheal mucosa due to overfilling the cuff. It is known that $20-30 \%$ of cuff pressure measurements may deviate above or below this threshold. ${ }^{1}$ However, the impact of such deviation on clinical outcomes is not well described in the medical literature. ${ }^{2}$ The 2 most common methods for assessing cuff pressures are using a manometer for direct measurement and performing a minimal leak, with the former being the most accepted method. ${ }^{3}$ However, the optimal frequency for conducting cuff pressure assessments is unknown. One study suggests that the act of measuring cuff pressures results in the removal of air from the cuff and can inadvertently result in underinflation of the cuff. ${ }^{4}$ Moreover, a recent animal study suggests that the material used to manufacture the endotracheal tube cuff may play a 
more important role in determining the presence or absence of mucosal injury, with polyurethane being tolerated better than polyvinylchloride. ${ }^{5}$

\section{See the Related Editorial on Page 632}

An important limitation of the current literature pertaining to the frequency of cuff pressure monitoring is the lack of clinical outcome assessment for various frequencies of monitoring. Given the lack of a consensus on the optimal frequency for monitoring cuff pressure, we performed a prospective trial to address this issue. The main goal of this study was to compare frequent cuff pressure monitoring to infrequent monitoring so that we could determine whether these 2 strategies resulted in identifiable differences in clinical outcomes, including respiratory-related complications.

\section{Methods}

\section{Study Population and Data Source}

This study was conducted within the 2 medical ICUs (34 beds) at Barnes-Jewish Hospital, an academic referral center of 1,250 beds in St. Louis, Missouri. The medical ICUs are geographically co-located closed units with shared physician, nursing, pharmacy, and respiratory therapy staffs. The medical ICUs are staffed at all times by these teams, including intensivists who are boardcertified in critical care. The Washington University School of Medicine Human Studies Committee approved this investigation, and the need for informed consent was waived (Institutional Review Board Number: 201605155; ClinicalTrials.gov Identifier: NCT02950519). All patients admitted to the medical ICUs from October 10, 2016, through February 28, 2017, who required endotracheal intubation and mechanical ventilation were eligible for inclusion. Subjects could only be enrolled once in this investigation. Patients with a tracheostomy were excluded. Data were prospectively collected from the electronic health record and from subjects' ICU teams.

\section{Study Outcomes/Objectives}

The primary outcome of this study was to determine whether and how frequently ventilator-associated events (VAEs) occurred. Secondary outcomes included 30-d and 100-d mortality, length of stay, witnessed aspiration events, ventilatorassociated pneumonia (VAP), tracheal complications, and 30-d hospital readmission. The primary outcome (VAEs) and the occurrence of VAP were directly assessed by the Hospital Epidemiology and Infection Prevention Department of Barnes-Jewish Hospital in a prospective manner. The infec-

\section{QUICK LOOK}

\section{Current knowledge}

There is limited available research to guide clinicians on the optimal frequency of performing endotracheal tube cuff pressure measurements. Current practices vary throughout the world, from very infrequent cuff pressure measurements to continuous assessments of cuff pressure.

\section{What this paper contributes to our knowledge}

Despite an almost 5-fold difference in cuff pressure assessments, we found no difference in the primary outcome of ventilator-associated events between the study groups. There were also no differences in the secondary outcomes, which included length of stay, mortality, and ventilator-associated pneumonia. Our study suggests that infrequent cuff pressure monitoring is safe and can be performed only as clinically indicated, thus allowing respiratory care resources to be directed to other potentially more crucial issues.

tion prevention department was blinded to the conduct of this study and to the implication of subject room assignments with regard to this investigation. Determination of VAEs and VAP are routinely carried out by the infection prevention department as part of their surveillance and quality improvement mandate, as well as for public reporting. This method of assessing VAEs has been used previously for clinical studies carried out at Barnes-Jewish Hospital.6,7 Moreover, the medical ICUs have a long history of routinely using protocols for the weaning of mechanical ventilation, sedation management, and prevention of VAP and VAEs developed in conjunction with the infection prevention department and the BarnesJewish Hospital Departments of Respiratory Care and Nursing. ${ }^{6,8-10} \mathrm{We}$ also recorded the exact number of endotracheal tube cuff pressure assessments performed in both groups.

\section{Definitions and Study Design}

All subjects admitted to the medical ICUs were assigned to receive either frequent or infrequent monitoring of their cuff pressures according to their room assignment. Subjects assigned by the hospital admitting office to an oddnumbered room received infrequent cuff pressure monitoring, which was defined as after tracheal intubation (if performed in the medical ICU) or upon ICU admission if endotracheal intubation occurred in the emergency department or on a hospital ward, and if there was a clinical indication to assess the cuff pressure, such as an audible cuff leak or loss of tidal volume. Subjects assigned by the hospital admitting office to an even-numbered room re- 
Table 1. Additional Criteria for the Definition of Ventilator-Associated Pneumonia*

\begin{tabular}{|c|c|}
\hline Criteria 1 & $\begin{array}{l}\text { Purulent respiratory secretions from } \geq 1 \text { specimen collections }+1 \text { of the following positive cultures } \dagger \text { : } \\
\text { - Endotracheal aspirate, } \geq 10^{5} \mathrm{CFU} / \mathrm{mL} \text { or equivalent semi-quantitative result } \\
\text { - Bronchoalveolar lavage, } \geq 10^{4} \mathrm{CFU} / \mathrm{mL} \text { or equivalent semi-quantitative result } \\
\text { - Lung tissue, } \geq 10^{4} \mathrm{CFU} / \mathrm{mL} \text { or equivalent semi-quantitative result } \\
\text { - Protected specimen brush, } \geq 10^{3} \mathrm{CFU} / \mathrm{mL} \text { or equivalent semi-quantitative result }\end{array}$ \\
\hline Criteria 2 & $\begin{array}{l}\text { One of the following (without the requirement for purulent respiratory secretions): } \\
\text { - Positive pleural fluid culture where the specimen was obtained during thoracentesis or initial placement of chest tube and } \\
\text { not from an indwelling chest tube } \\
\text { - Positive lung histopathology } \\
\text { - Positive diagnostic test for Legionella species } \\
\text { - Positive diagnostic test on respiratory secretions for influenza virus, respiratory syncytial virus, adenovirus, parainfluenza } \\
\text { virus, rhinovirus, human metapneumovirus, or coronavirus }\end{array}$ \\
\hline \multicolumn{2}{|c|}{$\begin{array}{l}\text { * Criteria } 1 \text { or } 2 \text { had to be met in addition to an abnormal white blood cell count or temperature and a new antibiotic start. } \\
\dagger \text { Excluding normal oral flora, Candida species or yeast not otherwise specified, coagulase-positive Staphylococcus species, and Enterococcus species. } \\
\text { CFU = colony-forming unit }\end{array}$} \\
\hline
\end{tabular}

ceived frequent cuff pressure monitoring, which was defined in the same manner as subjects assigned to oddnumbered rooms with the addition of a cuff pressure assessment at the beginning of every 8-h shift (ie, 3 times daily). All cuff pressure assessments were carried out by credentialed respiratory therapists with a manometric syringe, with a targeted pressure of $24-30 \mathrm{~cm} \mathrm{H}_{2} \mathrm{O}$ without evidence of an audible air leak or loss of tidal volume (AG Cuffill, Hospitech Respiration, Petach Tikva, Israel).

The definitions used for VAEs and VAP were taken from the recently published update from the Centers for Disease Control and Prevention. ${ }^{11}$ To meet the VAE definition, a subject who was mechanically ventilated must have had at least $2 \mathrm{~d}$ of stable or decreasing daily minimum PEEP or $\mathrm{F}_{\mathrm{IO}_{2}}$ followed by at least $2 \mathrm{~d}$ of increased daily minimum PEEP or $\mathrm{F}_{\mathrm{IO}_{2}}$, in which the increase in the daily minimum PEEP is $\geq 3 \mathrm{~cm} \mathrm{H}_{2} \mathrm{O}$ or the increase in the daily minimum $\mathrm{F}_{\mathrm{IO}_{2}}$ is $\geq 0.20$ (or $20 \%$ in oxygen concentration). VAP was defined by the presence of an abnormal white blood cell count $\left(\geq 12,000\right.$ cells $/ \mathrm{mm}^{3}$ or $\leq 4,000$ cells $\left./ \mathrm{mm}^{3}\right)$ or temperature $\left(>38^{\circ} \mathrm{C}\right.$ or $\left.<36^{\circ} \mathrm{C}\right)$ and a new antimicrobial start for that indication. In addition, subjects assessed as having VAP had to meet the criteria demonstrated in Table 1. A witnessed aspiration event required the aspiration event to be observed by one of the subject's health care providers and documented in the medical record. Only the first episodes of VAE and VAP were collected as outcomes.

Thirty-day hospital readmission, 30-d mortality, 100-d mortality, and tracheal complications were assessed using the BJC Healthcare Informatics database. Barnes-Jewish Hospital is the main teaching institution in a large integrated health care system of both in-patient and out-patient care. The system includes 13 hospitals in a compact geographic region surrounding and including St. Louis, Missouri. Barnes-Jewish Hospital has $>50,000$ admissions annually, and the BJC system $>140,000$. We included readmission to any of these hospitals in our analysis. People treated within this health care system are, in nearly all cases, readmitted to one of the system's participating 13 hospitals. If a patient who receives health care in the system presents to a non-system hospital, he/she is often transferred back into the integrated system because of issues of insurance coverage. Similarly, we examined the available out-patient and in-patient records of all study subjects to identify any tracheal complications recorded for up to $100 \mathrm{~d}$ after study entry.

\section{Endotracheal Tube and ICU Protocols}

All subjects enrolled in this study were intubated with a Shiley Hi-Lo Oral/Nasal Tracheal Tube Cuffed (Covidien, Dublin, Ireland), utilizing a size 7.0, 7.5, 8.0, or 8.5 tube. The Barnes-Jewish Hospital protocol requires patients to be ventilated using the assist-control mode with a 980 Puritan-Bennett 840 ventilator (Medtronic, Minneapolis, $\mathrm{MN})$. Historically in these ICUs, mechanical ventilation, weaning of mechanical ventilation, as well as the use of sedation, are standardized according to the protocols established for Barnes-Jewish Hospital.6,8-10

\section{Statistical Analyses}

We expected that, with a sample size of 292 subjects (146 in each group), we would be able to demonstrate non-inferiority between the 2 groups assuming VAE rates of $8 \%$ in the infrequent monitoring group and $5 \%$ in the frequent monitoring group, respectively, and assuming a 2 -sided $\alpha$ error of 0.05 and a power of $95 \%$ with a $\Delta$ value of $12.5 \% .^{6,12}$ Continuous variables were expressed as means \pm SDs or medians and interquartile range when appropriate. The $t$ test was used to analyze normally distrib- 


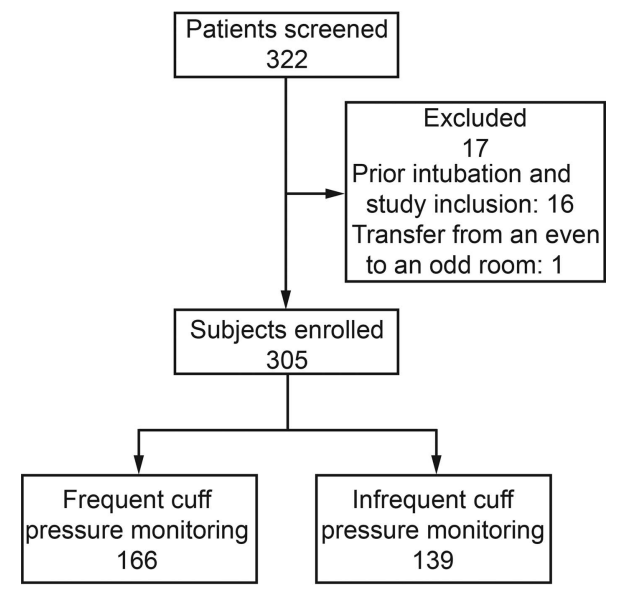

Fig. 1. Flow chart.

uted continuous variables, whereas the Mann-Whitney $U$ test was used to analyze non-normally distributed continuous variables. Categorical data were reported as frequency distributions and analyzed using the chi-square test. Cumulative survival was compared between groups with Kaplan-Meier curves. $P$ values $<.05$ were considered statistically significant, and all tests were 2-tailed. All analyses were done using SPSS Statistics 21 (IBM SPSS Statistics, version 21.0. Armonk, New York).

\section{Results}

During the study period, we screened 322 consecutive intubated patients admitted to the medical ICUs (Fig. 1). We excluded 17 patients, leaving a total of 305 subjects in the study cohort. The reasons for patient exclusion included prior intubation and inclusion in the study $(n=16)$ and transfer from an even-numbered room to an odd-numbered room with a change in cuff pressure monitoring $(n=1)$.

Among the 305 intubated subjects, 166 (54.4\%) were assigned to frequent cuff pressure monitoring, and 139 (44.6\%) were assigned to infrequent cuff pressure monitoring. The total numbers of cuff pressure monitoring events for these groups were 1,531 and 336, respectively. Table 2 shows the baseline characteristics of the study population. Demographics and severity of illness according to Acute Physiology and Chronic Health Evaluation II scores were similar for both groups. Subjects assigned to the frequent monitoring group were more likely have an underlying malignancy compared to subjects in the infrequent monitoring group. The reasons for intubation and mechanical ventilation were also similar for the 2 groups. The most common reasons for tracheal intubation in both groups were for airway protection, shock, pneumonia, the conduct of a procedure such as endoscopy, and pulmonary edema (Table 2).

The occurrence of VAEs was infrequent and similar for both groups (3.6\% vs 5.8\%, $P=.37$ ) (Table 3 ). Witnessed aspiration events $(0.6 \%$ vs $0 \%, P=.36)$, VAP (0\% vs $0.7 \%, P=.27)$, 30-d mortality (31.3\% vs $30.2 \%, P=.83)$, and median length of hospital stay (10 d [interquartile range 6-21 d] vs $11 \mathrm{~d}$ [interquartile range 6-21d], $P=.34$ ) were also similar for both study groups. The 30-d hospital readmission rate was statistically less for the group receiving infrequent endotracheal tube cuff pressure monitoring (6.5\% vs $15.1 \%, P=.02$ ). Kaplan-Meier curves comparing survival showed no difference for subjects in the frequent monitoring group compared to the infrequent monitoring group (Fig. 2).

\section{Discussion}

We found that more frequent monitoring of cuff pressures with a nearly 5-fold difference in the number of monitoring episodes between study groups, was not associated with any demonstrable clinical outcome benefit. The rates of VAEs and VAP were relatively low but similar between both study groups. We also found that ICU and hospital stay did not differ by group assignment, and the number of witnessed aspiration events was exceedingly low. We did observe a statistically greater rate of 30-d hospital readmission among subjects assigned to receive more frequent cuff pressure monitoring. This study represents the largest trial to date that compares frequent monitoring of cuff pressure with infrequent monitoring. These findings have potential implications in terms of the frequency with which routine cuff pressure monitoring should occur in the ICU setting.

A recent survey of intensivists in Queensland, Australia, and an accompanying systematic literature review of cuff pressure monitoring found that the majority of respondents (28 of 29) routinely monitor tracheal cuff function, primarily using manometric cuff pressure measurements. ${ }^{13}$ However, these same investigators found that the targeted cuff pressures varied, with 3 of 26 respondents aiming for $10-20 \mathrm{~cm} \mathrm{H}_{2} \mathrm{O}, 10$ of 26 aiming for $21-25 \mathrm{~cm} \mathrm{H}_{2} \mathrm{O}$, and 13 of 26 aiming for $26-30 \mathrm{~cm} \mathrm{H}_{2} \mathrm{O}$. Fifteen of the 29 respondents also reported that they had no current guideline or protocol for tracheal cuff management, and only 16 of 29 indicated that there was a dedicated area in the clinical record for reporting cuff interventions. The findings of this survey suggest that there is significant variability in the way cuff pressures are monitored in the hospital setting. Similarly, another meta-analysis that examined various methods for assessing cuff pressures, including objective measurement as compared with subjective assessments alone, found significant differences between the 2 methods. ${ }^{14}$ These included presumed benefits with objective measurement including less cough, hoarseness, and sore throat after extubation, fewer lesions of the trachea, and silent aspiration, as well as maintaining accurate cuff pressures. However, neither of these analyses was able to 
Table 2. Baseline Characteristics

\begin{tabular}{|c|c|c|c|}
\hline & $\begin{array}{l}\text { Frequent Cuff Pressure } \\
\text { Monitoring }(n=166)\end{array}$ & $\begin{array}{l}\text { Infrequent Cuff Pressure } \\
\text { Monitoring }(n=139)\end{array}$ & $P$ \\
\hline Age, median (IQR) & $60(46-67)$ & $60(47-70)$ & .68 \\
\hline \multicolumn{4}{|l|}{ Sex, $n(\%)$} \\
\hline Male & $97(58.4)$ & $85(61.2)$ & \multirow[t]{2}{*}{.63} \\
\hline Female & 69 (41.6) & $54(38.8)$ & \\
\hline \multicolumn{4}{|l|}{ Race, $n(\%)$} \\
\hline Caucasian & 89 (53.6) & $84(60.4)$ & \multirow[t]{3}{*}{.28} \\
\hline African American & $67(40.4)$ & $51(36.7)$ & \\
\hline Other & $10(6.0)$ & $4(2.9)$ & \\
\hline APACHE II score, median (IQR) & $14(9-21)$ & $14(10-21)$ & .56 \\
\hline \multicolumn{4}{|l|}{ Comorbidities, $n(\%)$} \\
\hline Cardiac & $61(36.7)$ & $45(32.4)$ & .42 \\
\hline Renal & $37(22.3)$ & $27(19.4)$ & .54 \\
\hline Dialysis & $9(5.4)$ & $4(2.9)$ & .27 \\
\hline Liver & $31(18.7)$ & $24(17.3)$ & .75 \\
\hline Solid organ transplant & $7(4.2)$ & $5(3.6)$ & .78 \\
\hline Hematopoietic stem cell transplant & $2(1.2)$ & $0(0)$ & .19 \\
\hline Malignancy & $46(27.7)$ & $25(18.0)$ & .045 \\
\hline HIV & $4(2.4)$ & $2(1.4)$ & .54 \\
\hline COPD & $38(22.9)$ & $32(23.0)$ & .98 \\
\hline \multicolumn{4}{|l|}{ Intubation reason, $n(\%)$} \\
\hline Airway protection & $63(38.0)$ & $56(40.3)$ & \multirow[t]{7}{*}{.59} \\
\hline COPD/asthma exacerbation & $7(4.2)$ & $9(6.5)$ & \\
\hline Pneumonia & $21(12.7)$ & $22(15.8)$ & \\
\hline Procedural & $23(13.9)$ & $11(7.9)$ & \\
\hline Pulmonary edema & $18(10.8)$ & $11(7.9)$ & \\
\hline Shock & $23(13.9)$ & $19(13.7)$ & \\
\hline Other* & $11(6.6)$ & $11(7.9)$ & \\
\hline \multicolumn{4}{|c|}{$\begin{array}{l}\text { * Includes pulmonary embolism, pleural effusion, obesity hypoventilation syndrome, interstitial lung disease, cystic fibrosis, and myasthenia gravis. } \\
\text { APACHE = Acute Physiology and Chronic Health Evaluation } \\
\text { IQR = interquartile range } \\
\text { HIV = human immunodeficiency virus }\end{array}$} \\
\hline
\end{tabular}

assess the impact of variations in the frequency of cuff pressure assessments due to a lack of available data.

Previous studies have demonstrated that ventilator circuits can be used safely without routine changing of the circuit. ${ }^{15-17}$ It has also been shown that in-line suction catheters $^{18,19}$ and heat and moisture exchangers ${ }^{20,21}$ can be used for more prolonged periods without increasing the risk of nosocomial infection or other adverse outcomes. Other clinical studies have found that more frequent manipulation of the ventilator circuit or its components can result in more frequent complications, including aspiration events and pneumonia. ${ }^{15,22}$ Moreover, various modifications to the design of endotracheal tubes have occurred to prevent the development of biofilms on the tube surface, tube obstruction, and aspiration events. ${ }^{23}$ These modifications have been developed due to concerns over routinely exchanging endotracheal tubes, such as desaturation, aspiration, and the development of pneumonia. ${ }^{23}$ Given the predominance of evidence that less frequent manipulation of the ventilator circuit and its components is safe and is potentially less injurious to the patient, it seems logical to extend this observation to the assessment of cuff pressure monitoring frequency. The lack of available data on how often cuff pressure monitoring should optimally occur served as the justification for this investigation. Not only does our study find a lack of clinical benefit with more frequent monitoring of cuff pressures, but it suggests that the efforts of respiratory therapists who perform frequent routine cuff pressure assessments could be better spent in other areas of patient care.

Several limitations of our study should be recognized. We did not routinely record the absolute values of the cuff pressure measurements, so we cannot be certain whether there were differences in the absolute values of the cuff pressure measurements between the study groups. This seems unlikely, however, given the lack of outcome differences in our study. In addition, we did not perform any supplemental exams of the larynx or trachea in the subjects, which might have identified early signs of airway injury. The choice to use room assignments in the study 
Table 3. Clinical Outcomes

\begin{tabular}{|c|c|c|c|}
\hline & $\begin{array}{l}\text { Frequent Cuff Pressure } \\
\text { Monitoring }(n=166)\end{array}$ & $\begin{array}{l}\text { Infrequent Cuff Pressure } \\
\text { Monitoring }(n=139)\end{array}$ & $P$ \\
\hline Ventilator-associated event, $n(\%)$ & $6(3.6)$ & $8(5.8)$ & .37 \\
\hline Ventilator-associated pneumonia, $n(\%)$ & $0(0)$ & $1(0.7)$ & .27 \\
\hline 30-d mortality, $n(\%)$ & $52(31.3)$ & $42(30.2)$ & .83 \\
\hline 100-d mortality, $n(\%)$ & $67(40.4)$ & $46(33.1)$ & .19 \\
\hline Endotracheal tube cuff pressure assessments, median (IQR) & $5(2-13)$ & $1(1-3)$ & $<.001$ \\
\hline Duration of mechanical ventilation, $d$, median (IQR) & $3(2-6)$ & $4(2-7)$ & .27 \\
\hline Length of stay in medical ICU, d, median (IQR) & $5(3-10)$ & $6(3-10)$ & .52 \\
\hline Hospital length of stay, d, median (IQR) & $10(6-21)$ & $11(6-21)$ & .34 \\
\hline LTAC/SNF transfer, $n(\%)$ & $36(21.7)$ & $40(28.8)$ & .15 \\
\hline 30-d readmission, $n(\%)$ & $25(15.1)$ & $9(6.5)$ & .02 \\
\hline Vasopressors, $n(\%)$ & $86(51.8)$ & $84(60.4)$ & .13 \\
\hline Tracheostomy, $n(\%)$ & $15(9.0)$ & $16(11.5)$ & .48 \\
\hline Tracheal stenosis, $n(\%)$ & $1(0.6)$ & $1(0.7)$ & .90 \\
\hline Re-intubated during hospitalization, $n(\%)$ & $12(7.2)$ & $10(7.2)$ & .91 \\
\hline Witnessed aspiration event, $n(\%)$ & $1(0.6)$ & $0(0)$ & .36 \\
\hline $\begin{array}{l}\mathrm{QQR}=\text { interquartile range } \\
\mathrm{LTAC}=\text { long-term acute care } \\
\mathrm{SNF}=\text { skilled nursing facility }\end{array}$ & & & \\
\hline
\end{tabular}

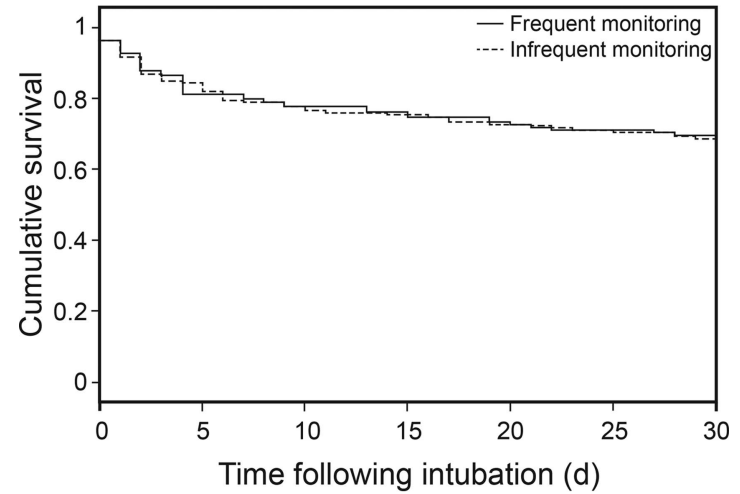

Fig. 2. Kaplan-Meier curves for cumulative survival for the group that had frequent endotracheal tube cuff pressure monitoring and the group that had infrequent monitoring (log-rank test $=0.84$ ).

design to allocate subjects into their respective study groups could allow for unblinded assessments of the primary outcome. To minimize this bias, we obtained all assessments of our primary outcome VAEs, as well as the occurrence of VAP, from the infection prevention department. The infection prevention nurses were blinded to the performance of this investigation and to the implications of room assignment on cuff pressure monitoring. Moreover, the assessments of VAEs and VAP were carried out from locations remote from the medical ICUs by using the hospital's information system. Because we only carried out this study in medical subjects, it is possible that our results might have varied if we included subjects from the other ICUs in our institution, such as surgical, trauma, neurology, cardiothoracic, and bone marrow transplant patients.
However, the lack of any identifiable signal suggesting a beneficial outcome with more frequent cuff pressure monitoring makes this unlikely. Moreover, the practice of cuff pressure monitoring is the same at our hospital for all patients receiving mechanical ventilation.

Another important limitation of our study is that we had a relatively small number of subjects with VAEs and VAP. This may be due in part to the staffing practices in the medical ICUs and the history of efforts directly aimed at optimizing the care of ventilated subjects in those units. ${ }^{6,8-10} \mathrm{How}-$ ever, we cannot be certain that our findings would be the same in ICUs having different staffing models and protocols for the prevention of events such as VAEs and VAP. Our study data were also derived from a single center, which limits the generalizability of our findings. As such, our results may not reflect what one might see at other institutions. Finally, the infection prevention department obtained VAE and VAP data primarily for benchmarking purposes. It is possible the reporting of these data were biased due to the methods used in their detection. This ascertainment bias would be the same for both treatment groups, however, so it is unlikely that this influenced our findings.

\section{Conclusions}

In summary, our study suggests that infrequent monitoring of cuff pressures is likely safe compared to more frequent monitoring. Many hospitals perform frequent routine monitoring of cuff pressures despite the absence of an audible leak or a loss of tidal volume. ${ }^{1,13,14}$ If the results of our study can be confirmed by other centers, then these data could instill 
confidence in clinicians to change this practice and only monitor cuff pressures after intubation, after manipulation or adjustment of the endotracheal tube, and when clinically indicated for an air leak or a loss of tidal volume. The era of performing frequent routine assessments of cuff pressures may be coming to an end, as has been suggested for the assessment of frequent gastric residual volumes, due to the lack of observed clinical benefit of routine monitoring. ${ }^{24,25}$ However, the need to confirm our results and the advent of new technologies that allow for the continuous monitoring of endotracheal tube cuff pressures may require further study to determine the potential clinical impact, or lack thereof, of such approaches. ${ }^{26}$

\section{REFERENCES}

1. Carhart E, Stuck LH, Salzman JG. Achieving a safe endotracheal tube cuff pressure in the prehospital setting: is it time to revise the standard cuff inflation practice? Prehosp Emerg Care 2016;20(2):273-277.

2. Danielis M, Benatti S, Celotti P, De Monte A, Trombini O. Continuous monitoring of endotracheal tube cuff pressure: best practice in intensive care unit. Assist Inferm Ric 2015;34(1):15-20.

3. Totonchi Z, Jalili F, Hashemian SM, Jabardarjani HR. Tracheal stenosis and cuff pressure: comparison of minimal occlusive volume and palpation techniques. Tanaffos 2015;14(4):252-256.

4. Motoyama A, Asai S, Konami H, Matsumoto Y, Misumi T, Imanaka $\mathrm{H}$, Nishimura M. Changes in endotracheal tube cuff pressure in mechanically ventilated adult patients. J Intensive Care 2014;2(1):7.

5. Li Bassi G, Luque N, Martí JD, Aguilera Xiol E, Di Pasquale M, Giunta V, et al. Endotracheal tubes for critically ill patients: an in vivo analysis of associated tracheal injury, mucociliary clearance, and sealing efficacy. Chest 2015;147(5):1327-1335.

6. Nolley EP, Trevino SE, Babcock HM, Kollef MH. A targeted educational intervention to reduce ventilator-associated complications. Am J Infect Control 2016;44(11):1406-1407.

7. Boyer AF, Schoenberg N, Babcock H, McMullen KM, Micek ST, Kollef MH. A prospective evaluation of ventilator-associated conditions and infection-related ventilator-associated conditions. Chest 2015;147(1):68-81.

8. Kollef MH, Shapiro SD, Silver P, St John RE, Prentice D, Sauer S, et al. A randomized, controlled trial of protocol-directed versus physician-directed weaning from mechanical ventilation. Crit Care Med 1997;25(4):567-574.

9. Brook AD, Ahrens TS, Schaiff R, Prentice D, Sherman G, Shannon W, Kollef MH. Effect of a nursing-implemented sedation protocol on the duration of mechanical ventilation. Crit Care Med 1999; 27(12):2609-2615.

10. Skrupky LP, Drewry AM, Wessman B, Field RR, Fagley RE, Varghese L, et al. Clinical effectiveness of a sedation protocol minimizing benzodiazepine infusions and favoring early dexmedetomidine: a before-after study. Crit Care 2015;19:136.

11. Magill SS, Klompas M, Balk R, Burns SM, Deutschman CS, Diekema $\mathrm{D}$, et al. Developing a new, national approach to surveillance for ventilator-associated events. Crit Care Med 2013;41(11):2467-2475.
12. Sealed Envelope Ltd. 2012. Power calculator for binary outcome noninferiority trial. https://www.sealedenvelope.com/power/binary-noninferior. Accessed May 17, 2016.

13. Talekar CR, Udy AA, Boots RJ, Lipman J, Cook D. Tracheal cuff pressure monitoring in the ICU: a literature review and survey of current practice in Queensland. Anaesth Intensive Care 2014;42(6):761-770.

14. Hockey CA, van Zundert AA, Paratz JD. Does objective measurement of tracheal tube cuff pressures minimise adverse effects and maintain accurate cuff pressures? A systematic review and metaanalysis. Anaesth Intensive Care 2016;44(5):560-570.

15. Craven DE, Kunches LM, Kilinsky V, Lichtenberg DA, Make BJ, McCabe WR. Risk factors for pneumonia and fatality in patients receiving continuous mechanical ventilation. Am Rev Respir Dis 1986;133(5):792-796.

16. Dreyfuss D, Djedaini K, Weber P, Brun P, Lanore JJ, Rahmani J, et al. Prospective study of nosocomial pneumonia and of patient and circuit colonization during mechanical ventilation with circuit changes every 48 hours versus no change. Am Rev Respir Dis 1991;143(4 Pt 1):738-743.

17. Kollef MH, Shapiro SD, Fraser VJ, Silver P, Murphy DM, Trovillion $\mathrm{E}$, et al. Mechanical ventilation with or without 7-day circuit changes: a randomized controlled trial. Ann Intern Med 1995;123(3):168174.

18. Kollef MH, Prentice D, Shapiro SD, Fraser VJ, Silver P, Trovillion E, et al. Mechanical ventilation with or without daily changes of in-line suction catheters. Am J Respir Crit Care Med 1997; 156(2 Pt 1):466-472.

19. Stoller JK, Orens DK, Fatica C, Elliott M, Kester L, Woods J, et al. Weekly versus daily changes of in-line suction catheters: impact on rates of ventilator-associated pneumonia and associated costs. Respir Care 2003;48(5):494-499.

20. Kollef MH, Shapiro SD, Boyd V, Silver P, Von Harz B, Trovillion E, Prentice D. A randomized clinical trial comparing an extendeduse hygroscopic condenser humidifier with heated-water humidification in mechanically ventilated patients. Chest 1998;113(3):759767.

21. Boisson C, Viviand X, Arnaud S, Thomachot L, Miliani Y, Martin C. Changing a hydrophobic heat and moisture exchanger after 48 hours rather than 24 hours: a clinical and microbiological evaluation. Intensive Care Med 1999;25(11):1237-1243.

22. Han J, Liu Y. Effect of ventilator circuit changes on ventilatorassociated pneumonia: a systematic review and meta-analysis. Respir Care 2010;55(4):467-474.

23. Haas CF, Eakin RM, Konkle MA, Blank R. Endotracheal tubes: old and new. Respir Care 2014;59(6):933-952.

24. Reignier J, Mercier E, Le Gouge A, Boulain T, Desachy A, Bellec F, et al. Effect of not monitoring residual gastric volume on risk of ventilator-associated pneumonia in adults receiving mechanical ventilation and early enteral feeding: a randomized controlled trial. JAMA 2013;309(3):249-256.

25. Rice TW. Gastric residual volume: end of an era. JAMA 2013; 309(3):283-284.

26. Lorente L, Lecuona M, Jiménez A, Lorenzo L, Roca I, Cabrera J, et al. Continuous endotracheal tube cuff pressure control system protects against ventilator-associated pneumonia. Crit Care 2014;18(2):R77.

This article is approved for Continuing Respiratory Care Education credit. For information and to obtain your CRCE

(free to AARC members) visit www.rcjournal.com

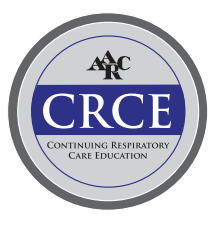

